\title{
Cenplestao
}

\section{Modelos de práticas informacionais}

\author{
Janicy Aparecida Pereira Rocha \\ Doutoranda; Universidade Federal de Minas Gerais (UFMG), Belo Horizonte, MG, Brasil; \\ janicy.rocha@gmail.com \\ Adriana Bogliolo Sirihal Duarte \\ Doutora; Universidade Federal de Minas Gerais (UFMG), Belo Horizonte, MG, Brasil; \\ bogliolo@eci.ufmg.br \\ Claudio Paixão Anastácio de Paula \\ Doutor; Universidade Federal de Minas Gerais (UFMG), Belo Horizonte, MG, Brasil; \\ claudiopap@hotmail.com
}

\begin{abstract}
Resumo: O trabalho apresenta e discute três modelos de práticas informacionais identificados na literatura da Ciência da Informação. A identificação ocorreu a partir de buscas realizadas no Portal de Periódicos da Coordenação de Aperfeiçoamento de Pessoal de Nível Superior e na Biblioteca Digital Brasileira de Teses e Dissertações. Todos os modelos foram desenvolvidos no contexto de busca de informação na vida cotidiana e adotam uma abordagem socioconstrucionista. Embora apresentem algumas semelhanças, os modelos diferem entre si por descreverem diferentes atividades e considerarem diferentes elementos como constituintes e/ou influenciadores das práticas informacionais identificadas.
\end{abstract}

Palavras-chave: Práticas informacionais. Comportamento informacional. Modelos. Busca de informação. Vida cotidiana.

\section{Introdução}

A compreensão de como o conceito de práticas informacionais vem constituindo-se no campo da Ciência da Informação está vinculada aos estudos de comportamento informacional (information behavior). Tais estudos, geralmente, abordam contextos de trabalho - profissionais como cientistas, engenheiros e outros - com o foco direcionado às dimensões cognitivas desses sujeitos. Assim, eles priorizam o comportamento individual em detrimento das interações presentes em comportamentos coletivos, concentrando-se na maneira como cada pessoa pensa, sente e age individualmente, em resposta às necessidades de informação. Além disso, nessas pesquisas, o contexto, 
geralmente, é abordado como um elemento que intervém nos pensamentos, nos sentimentos e nas ações dos sujeitos.

A partir da década de 1990, as demandas colocadas pelo surgimento da abordagem social dos estudos de usuários da informação constituem um cenário no qual o conceito de práticas informacionais tem conquistado espaço como uma alternativa crítica ao conceito de comportamento informacional. Sua adoção está vinculada às interações estabelecidas entre sujeitos e informação, em situações nas quais a informação e o conhecimento não são meramente cumulativos (ARAÚJO, 2013); nem resposta imediata a um estímulo, mas construídos coletiva e socialmente, de forma contínua, por sujeitos ativos.

Conforme o entendimento de Coimbra (2008), as pesquisas sobre comportamento informacional resultam, quase sempre, em modelos que, apesar de relevantes, não são capazes de abarcar a totalidade dos fenômenos possíveis na interação entre sujeitos e informação. Essas pesquisas deixam livre "[...] uma zona fenoménica, em parte conhecida, e possivelmente em grande parte por conhecer." (COIMBRA, 2008, p. 28) por meio dos estudos de práticas informacionais. Dito isso, entende-se que estudos de comportamento informacional e estudos de práticas informacionais têm o objetivo comum de compreender a interação entre sujeitos e informação. Entretanto, essa compreensão ocorre de forma distinta: os estudos de comportamento informacional têm o foco direcionado ao sujeito cognitivo, em detrimento da comunidade social, que é o foco dos estudos de práticas informacionais (HARLAN, 2012; WILSON; SAVOLAINEN, 2009).

Apesar dessas diferenças, alguns estudos de práticas informacionais também resultam em modelos. Alguns modelos de comportamento informacional já foram revisados por Martínez-Silveira e Oddone (2007) e por Furtado e Alcará (2015). Já esse trabalho tem como objetivo mapear e analisar os modelos de práticas informacionais existentes na literatura, bem como estabelecer eventuais relações entre eles.

\section{Modelos: definição}


Ao discutir o papel dos modelos no ambiente científico, Batista, Salvi e Lucas (2011) concluem que, nesse contexto, as noções de modelos e teorias apresentam estreita relação e, não raro, são ingenuamente confundidas. Eles ainda evidenciam dois pontos de vista sobre essa relação. O primeiro ponto de vista entende que modelos são elementos estruturantes de teorias; ambos são complementares, pois cada relação entre dois ou mais elementos da teoria deve ter relação correspondente entre dois ou mais elementos do modelo. O segundo ponto de vista entende que modelos são interpretações de teorias ou explicações sobre elas e, em alguns casos, podem ser ambas. Entretanto, modelos são mais restritos que teorias. Enquanto essas podem explicar fenômenos partindo de casos particulares até chegar a generalizações, aqueles são mais circunscritos e possibilitam a aplicação de princípios gerais das teorias em diferentes casos (BATISTA; SALVI; LUCAS, 2011).

Sayão (2001) aborda os modelos enquanto instrumentos de abstração úteis para a aquisição de conhecimento e para a representação e compreensão da realidade em áreas voltadas para fenômenos informacionais. Assim, “[...] um modelo é uma criação cultural, um 'mentefato', destinada a representar uma realidade, ou alguns dos seus aspectos, a fim de torná-los descritíveis qualitativa e quantitativamente e, algumas vezes, observáveis.” (SAYÃO, 2001, p. 83).

Por sua vez, Gouveia Júnior (1999) pontua três características dos modelos: (1) a redutividade refere-se ao número finito de elementos que são representados; (2) a pontualidade refere-se ao fato de que modelos representam um fato específico e isolado dos demais (3) o Zeitgeist, palavra de origem alemã, refere-se ao "[...] conjunto de crenças e elementos que determinam a visão de mundo de determinado indivíduo ou classe dentro de um contexto social e histórico específico.” (GOUVEIA JÚNIOR, 1999, p. 14).

A partir do exposto, os modelos podem ser entendidos como representações simplificadas, por meio das quais determinados aspectos da realidade são vislumbrados e melhor entendidos. Assim, são esquemas que representam a realidade de forma aproximada e, não raro, expressam aspectos da realidade conforme a visão de mundo de seu autor. Por serem criações humanas, 
modelos são constituídos por elementos significativos para quem os construiu e, portanto, elementos considerados secundários podem não ser incluídos.

Sayão (2001) afirma que os modelos que representam interações entre usuários e sistemas são denominados modelos conceituais e Coimbra (2008) entende que as pesquisas sobre comportamento informacional, geralmente, resultam em modelos desse tipo. Ainda para essa última autora, os modelos de comportamento informacional são compostos por um conjunto de categorias e um esquema de relações entre elas e têm objetivo descritivo e/ou explicativo. Enquanto os modelos descritivos dispõem, sequencialmente, um conjunto de categorias e conceitos; os modelos explicativos oferecem explicações teóricas a partir de relações sistemáticas entre conceitos (COIMBRA, 2008).

Ao contrário dos estudos acerca do comportamento informacional, os estudos de práticas informacionais não resultam, de forma significativa, em modelos. Percebe-se, na literatura sobre a temática (BØYUM; AABØ, 2015; COIMBRA, 2008; ISAH, 2009; NUNES, 2014; OLIPHANT, 2010), a prevalência de análises qualitativas, geralmente a partir da criação de categorias. Entretanto, existem, ainda que de modo incipiente, estudos de práticas informacionais nos quais as categorias e as relações entre elas são organizadas em forma de modelos descritivos ou explicativos.

\section{O campo de pesquisa de práticas informacionais}

Práticas informacionais é um conceito guarda-chuva considerado por Savolainen (2007) como adequado para investigar e descrever fenômenos relacionados à busca, ao uso e ao compartilhamento da informação. Fatores contextuais e sociais influenciam esses fenômenos e são abordados de forma distinta daquela dos estudos sobre comportamento informacional. O contexto é considerado como um elemento constitutivo das ações dos sujeitos e, ao mesmo tempo, por elas constituído a partir de uma relação dialógica. O individual e o social também são considerados como interdependentes.

Para Isah (2009), as pesquisas sobre práticas informacionais tendem a compreender os processos informacionais influenciados por dimensões 
socioculturais, bem como pelas relações dialógicas presentes no contexto em que tais práticas ocorrem. Segundo a autora, os estudos de comportamento informacional voltam-se para as ações de uma única pessoa realizando uma tarefa específica. Já a perspectiva de práticas informacionais desloca o foco de ações, motivações e habilidades individuais para práticas coletivas situadas em contexto. Tais práticas podem ser motivadas por necessidades ou demandas de informação, mas nem sempre se originam de uma tarefa estritamente definida.

Desse modo, entende-se que o principal ponto de distinção entre comportamento informacional e práticas informacionais é que essas compõem "[...] uma linha de investigação mais orientada sociologicamente e contextualmente [...]" (TALJA, 2005, p. 123, tradução nossa), enquanto aquele entende que as interações entre sujeitos e informação são desencadeadas por motivos e necessidades, muitas vezes individuais (SAVOLAINEN, 2007). Assim, os estudos de comportamento informacional abordam o sujeito cognitivo individual em situações nas quais a busca de informação é motivada pela necessidade informacional. Esses estudos, centrados em contextos científicos e/ou profissionais, como dito anteriormente, não contemplavam o contexto da vida cotidiana, tampouco situações como o encontro casual de informação ou buscas de informação sem a consciência de uma lacuna informacional, por exemplo.

O surgimento do conceito de práticas informacionais teria sido motivado, então, por essa necessidade de abordar a vida cotidiana, um contexto permeado por elementos diversos daqueles contemplados pelas pesquisas até então. Assim, os estudos de práticas informacionais são influenciados por um paradigma socioconstrucionista, no qual os processos de busca, uso e compartilhamento da informação são entendidos como práticas sociais instituídas em um domínio ou comunidade (HARLAN, 2012; MCKENZIE, 2003; SAVOLAINEN, 2007).

Ao elaborar um panorama das características contextuais referentes à necessidade de informação em estudos sobre busca de informação, Savolainen (2012) identificou três principais situações nas quais a necessidade de informação pode surgir: (1) situação da ação, (2) desempenho da tarefa e (3) diálogo. Na situação da ação, a necessidade informacional emerge a partir de 
uma situação e pode variar conforme fatores temporais, espaciais e situacionais. No desempenho da tarefa, a necessidade informacional surge a partir de uma tarefa a ser realizada ou de um problema a ser resolvido e o sucesso da busca de informação pode ser determinante para a conclusão da tarefa. Já o diálogo tem origem nas comunicações escritas ou verbais entre dois ou mais sujeitos, e a necessidade informacional é um entendimento construído conjuntamente, por meio de negociação discursiva.

Essas três situações, colocadas por Savolainen (2012), podem ser observadas tanto no contexto científico/profissional quanto no contexto da vida cotidiana, embora uma análise histórica dos estudos de usuários demonstre a tendência de que os estudos de comportamento informacional de abordagem cognitiva delimitem-se, majoritariamente, no contexto da tarefa. A observação de que esse não é o único contexto em que ocorre o relacionamento do indivíduo com a informação é que possibilita o surgimento do conceito de práticas informacionais e a expansão ou alargamento dos contextos de pesquisa, com a inclusão da situação da ação e do diálogo.

Atualmente, conforme Isah (2009) e Harlan (2012), as pesquisas sobre práticas informacionais têm sido desenvolvidas em quatro principais contextos, nos quais elas manifestam-se de diferentes formas. No ambiente de trabalho (workplace), são incluídos hospitais, hidrelétricas e outras organizações. No ambiente acadêmico (academic), são considerados laboratórios de pesquisa, universidades e escolas primárias. A aprendizagem no local de trabalho (worplace learning) inclui hospitais universitários, por exemplo. Por fim, a vida cotidiana (everyday life) abrange usuários de informação em saúde, usuários de redes sociais, donos de casa, imigrantes e outros. Savolainen (1995) foi o pioneiro a propor um modelo de busca de informação na vida cotidiana (Everyday Life Information Seeking, ELIS), apresentado a seguir.

\section{Busca de informação na vida cotidiana, por Reijo Savolainen}

Em meados da década de 1990, algumas questões relacionadas à busca e ao uso da informação começaram a ser reelaboradas, visando à superação da 
abordagem cognitiva presente em modelos de comportamento informacional. Dentre as iniciativas desenvolvidas com esse objetivo, destaca-se a pesquisa de Reijo Savolainen, voltada para a busca de informação não relacionada ao trabalho (nonwork information seeking). Conforme Savolainen (2006), a primeira motivação para o estudo referia-se à necessidade de abordar fatores sociais, culturais e psicológicos que influenciam as pessoas na preferência e no uso de determinadas fontes de informação em situações cotidianas. A segunda motivação voltava-se para o desenvolvimento de questões terminológicas para estudos de busca de informação e para a necessidade de enfatizar a busca de informação na vida cotidiana em relação à busca de informação relacionada ao trabalho (job-related information seeking). Savolainen (1995) esclarece que busca de informação na vida cotidiana e busca de informação relacionada ao trabalho são consideradas complementares, embora o modelo proposto por ele, apresentado na Figura 1, não destaque essa última.

Figura 1 - Modelo de busca de informação na vida cotidiana.

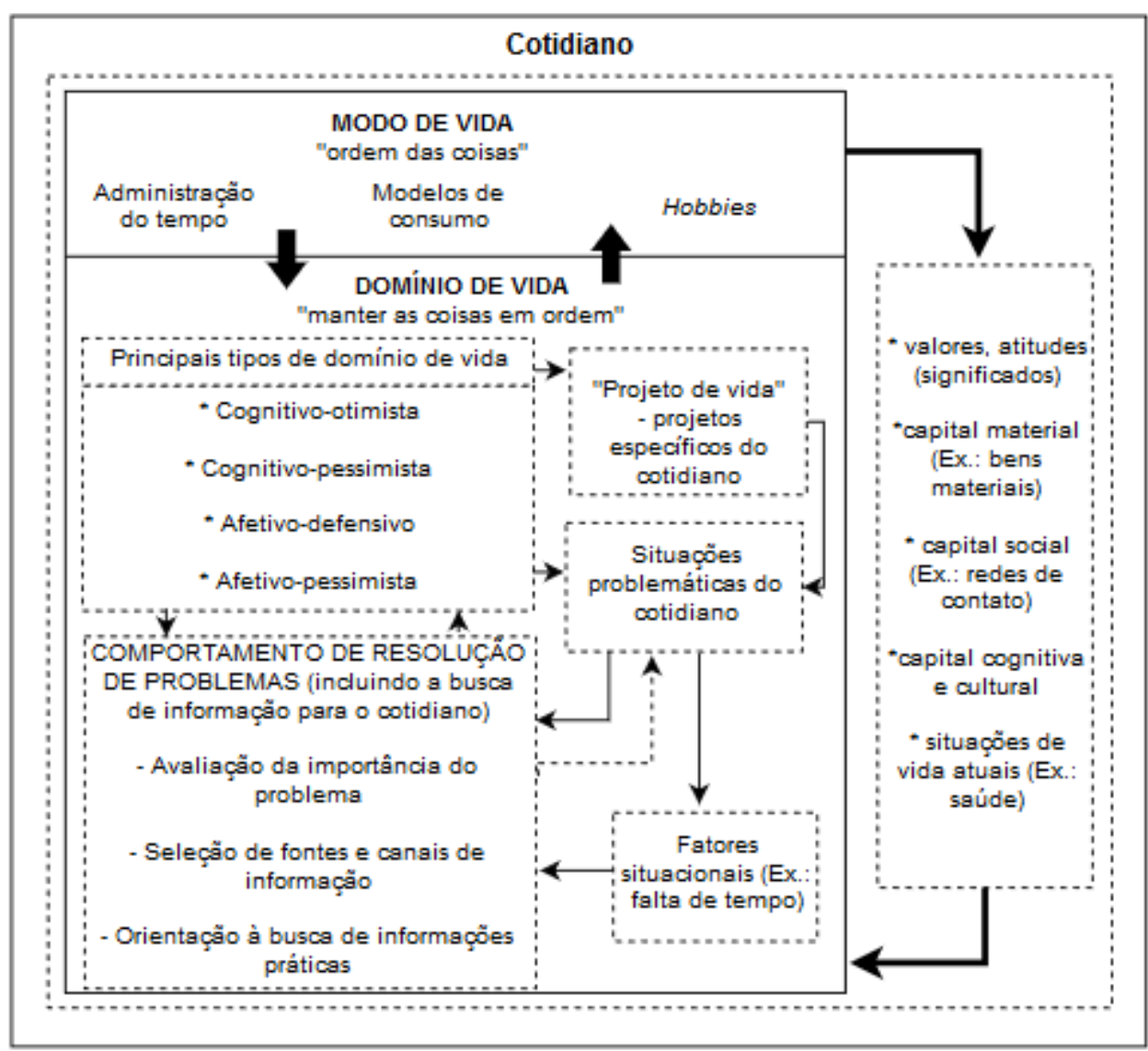

Fonte: Adaptado de Savolainen (1995, tradução nossa). 
O modelo parte de dois conceitos principais: (1) modo de vida (way of life) e (2) domínio da vida (mastery of life), ambos considerados como o contexto básico no qual as questões voltadas para a busca de informação não relacionada ao trabalho são examinadas. O modo de vida é o elemento por meio do qual são tratados os fatores sociais e culturais abordados pelo modelo. Sua definição é respaldada pela Teoria do Habitus (BOURDIEU, 1984), segundo a qual "[...] habitus pode ser definido como um sistema socialmente e culturalmente determinado de pensamento, percepção e avaliação, internalizado pelo indivíduo." (SAVOLAINEN, 1995, p. 261-262). Assim, o autor afirma que o habitus orienta as escolhas cotidianas e, portanto, o modo de vida representado pela ordem das coisas (orders of things) - é a manifestação prática do habitus. A ordem das coisas refere-se às escolhas feitas pelos sujeitos acerca das atividades cotidianas e é operacionalizada pela administração do tempo entre trabalho e lazer; pelos modelos de consumo relacionados a bens e serviços e pelos hobbies (SAVOLAINEN, 1995).

O domínio da vida consiste na preparação para resolver os problemas cotidianos, em conformidade com os próprios valores, visando manter as coisas em ordem (keeping things in order). A busca de informação tem papel fundamental nesse processo por auxiliar na resolução dos problemas. Quatro tipos de domínio da vida são apresentados por Savolainen (1995). O domínio da vida cognitivo-otimista (optimistic-cognitive) é caracterizado por uma forte confiança em resultados positivos para a resolução de problemas. O domínio da vida cognitivo-pessimista (pessimistic-cognitive) entende que existem problemas que não podem ser resolvidos de maneira otimista ainda que o sujeito busque informações de forma sistemática. O domínio da vida afetivo-defensivo (defensive-affective) fundamenta-se em visões otimistas sobre resolução de problemas; contudo, os sujeitos tendem a evitar fatores que evidenciam risco de falha. O domínio da vida afetivo-pessimista (pessimistic-affective) é composto por sujeitos que não confiam em suas habilidades para resolver os problemas cotidianos e evitam esforços para mudar essa situação.

Conforme representado no modelo, esses domínios da vida influenciam e são influenciados pelo comportamento de resolução de problemas dos sujeitos, 
que acontece por meio de três etapas: (1) avaliação da importância do problema; (2) seleção de fontes e canais de informação e (3) orientação à busca de informações práticas. Projeto de vida, situações problemáticas do cotidiano e fatores situacionais são elementos que direcionam a busca de informação no domínio da vida. Modo de vida e domínio da vida influenciam-se mutuamente e são afetados por valores, atitudes e situações de vida atuais, bem como por fatores sociais, culturais e individuais que são propriedades dos sujeitos. Esse conjunto de fatores inclui três tipos de capitais, a saber: (1) capital material, representado por bens materiais; (2) capital social, representado por redes de contato e (3) capital cultural, representado por recursos cognitivos adquiridos pela educação e pela experiência de vida.

A partir do modelo elaborado, Savolainen (1995) conduziu um estudo empírico baseado em entrevistas temáticas com dois grupos de sujeitos finlandeses - professores e industriais - visando compreender e comparar suas atividades de busca de informação na vida cotidiana. Tal escolha foi justificada por uma suposta diferença nos níveis educacionais e nos capitais social e cultural dos sujeitos de cada grupo. Os industriais finlandeses eram considerados a classe trabalhadora com menor nível educacional e os professores, a classe média com nível educacional maior. Os resultados mostraram que os modos de vida dos participantes de um grupo diferiam substancialmente do outro. Todavia, dentro do mesmo grupo também houve variações. Participantes pertencentes à mesma classe social, com nível de educação similar, condições de trabalho semelhantes e hobbies parecidos, comportavam-se de formas diferentes na busca de informação.

Embora o modelo de Savolainen (1995) não seja efetivamente um modelo de práticas informacionais, ele é um precursor desses, visto que chama a atenção para questões terminológicas e conceituais não consideradas pelos modelos de comportamento informacional. Suas principais contribuições para os atuais estudos de práticas informacionais são a noção de vida cotidiana e a percepção de que fatores sociais, culturais, individuais e temporais influenciam a resolução de problemas pelos sujeitos e a relação deles com a informação. 


\section{Metodologia}

Os modelos apresentados nesse trabalho foram identificados a partir de buscas realizadas em fevereiro de 2016, no Portal de Periódicos da Coordenação de Aperfeiçoamento de Pessoal de Nível Superior (CAPES) e na Biblioteca Digital Brasileira de Teses e Dissertações (BDTD). A escolha por essas bases de dados deve-se ao fato de o Portal Capes disponibilizar extenso acervo da produção científica nacional e internacional em formatos diversos e a BDTD agregar teses e dissertações produzidas nas diversas instituições de ensino nacionais.

Foram usadas as palavras-chaves "information practices" and "model" (em uma única expressão com o operador booleano “and") e "model of information practices", bem como suas correspondentes em português "práticas informacionais" and "modelos" e "modelos de práticas informacionais". Nenhum recorte cronológico ou idiomático foi estabelecido. Título, resumo e seções dos documentos recuperados foram analisados para verificar se, de fato, apresentavam algum modelo de práticas informacionais. Ao fim desse processo, três modelos de práticas informacionais foram identificados e, ordenados pelo ano de publicação, são apresentados nas próximas três seções.

\section{Modelo bidimensional de práticas informacionais, por Pamela McKenzie}

McKenzie (2003) investigou as necessidades de informação e as práticas informacionais de mulheres grávidas de gêmeos, bem como as fontes de informação usadas por elas. A perspectiva adotada considera o conceito de vida cotidiana - proposto por Savolainen (1995) - e o relaciona a uma situação específica (gravidez de gêmeos) vivida pelas participantes da pesquisa. Algumas questões principais foram exploradas: as formas ativas e acidentais de busca de informação; os papéis das diferentes fontes de informação (p. ex.: médicos e outras mães de gêmeos); a forma como as mulheres classificam as fontes de informação como confiáveis e úteis e as formas como elementos temporais da gravidez foram relacionados à busca de informação.

A autora considera que os modelos de comportamento informacional existentes são limitados para a compreensão da busca de informação na vida 
cotidiana por três principais motivos. Em primeiro lugar, tais modelos concentram-se na busca ativa e negligenciam práticas menos dirigidas de busca de informação. Em segundo lugar, esses modelos derivam de estudos em ambientes acadêmicos ou profissionais e, embora sejam úteis na descrição de buscas de informação sistemáticas nesses ambientes, eles não possibilitam uma compreensão holística da variedade do comportamento informacional na vida cotidiana. Finalmente, em terceiro lugar, muitos desses modelos enfatizam processos cognitivos individuais e, portanto, não permitem o entendimento de que a informação é construída por meio da interação entre indivíduo e contexto.

A partir de então, McKenzie (2003) defende que o encontro casual de informação na vida cotidiana tem importância equivalente à busca ativa e que uma abordagem construcionista para análise de discurso das participantes facilita a compreensão da construção social da informação. Assim, por meio da análise dos discursos das participantes, foram identificadas explicações complexas dos motivos pelos quais uma determinada fonte de informação apresentava maior ou menor autoridade que outra.

A partir das críticas aos modelos de comportamento informacional e do foco social proposto em sua pesquisa, McKenzie (2003) considera que o conceito de práticas informacionais é mais adequado ao contexto da vida cotidiana. Dessa maneira, considerando tal postura e os resultados obtidos por meio de entrevistas semiestruturadas em profundidade com 19 mulheres canadenses grávidas de gêmeos ${ }^{1}$, McKenzie (2003) elaborou o modelo bidimensional das práticas informacionais, apresentado na Figura 2.

No modelo são descritos quatro modos de busca de informação: (1) busca ativa (active seeking); (2) varredura ativa (active scanning); (3) monitoramento não-dirigido (non-directed monitoring) e (4) por procuração (by proxy). O modelo bidimensional contempla ainda duas etapas de busca de informação: (1) conexão (connecting) e (2) interação (interacting). Os quatro modos de busca não são, necessariamente, sequenciais; já as duas etapas são, uma vez que uma interação sempre é precedida pela conexão. 
Figura 2 - Modelo bidimensional de práticas informacionais.

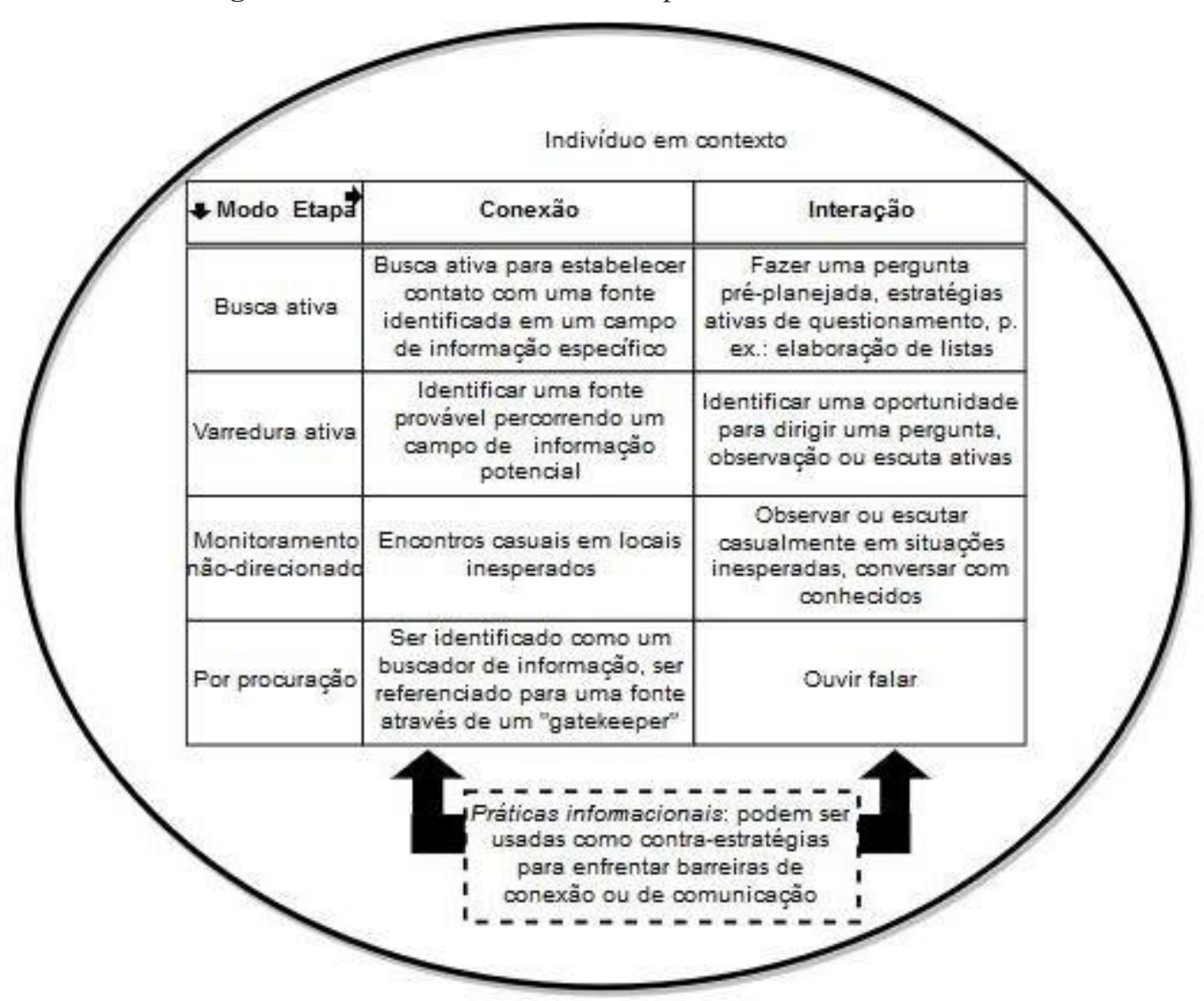

Fonte: Adaptado de McKenzie (2003, tradução nossa).

A autora considera a busca ativa como o modo mais direcionado das práticas informacionais, por meio do qual perguntas planejadas e pesquisas por itens conhecidos são feitas em fontes previamente conhecidas. Na varredura ativa, fontes prováveis como livrarias, consultórios médicos e grupos de discussão sobre o assunto desejado são exploradas. $\mathrm{O}$ monitoramento nãodirigido resulta na identificação acidental ou casual da informação, conforme o conceito de serendipity, adotado por McKenzie (2003) e entendido por Araújo (2015, doc. não paginado) como a possibilidade de "[...] encontrar determinados recursos informacionais relevantes sem estar procurando por eles.”. A busca por procuração, por sua vez, ocorre quando a interação com as fontes de informação acontece por meio de um intermediário. Na etapa de conexão, a fonte é identificada e o contato é estabelecido. Já na etapa de interação, o sujeito informacional interage com a fonte escolhida. 
As etapas, assim como os modos de busca de informação, são marcadas por práticas informacionais diversas. $\mathrm{Na}$ etapa de conexão, as participantes não só relataram práticas relacionadas ao processo de identificar fontes potenciais e estabelecer contato com elas, mas também apontaram barreiras capazes de restringir esses processos. Na etapa de interação, as práticas informacionais estavam relacionadas a estratégias para enfrentar as barreiras, como formular questões de pesquisa diretas ou perseverar na busca. O modelo também considera as dimensões casuais envolvidas no processo de busca de informação.

\section{Versão estendida do modelo de McKenzie, por Alison Yeoman}

Yeoman (2010) investigou as práticas informacionais de 35 mulheres inglesas na menopausa, com o intuito de relacionar os resultados encontrados ao modelo bidimensional de McKenzie (2003). O objetivo da autora era verificar a flexibilidade de aplicação do modelo em outro contexto. Os resultados obtidos por meio de entrevistas semiestruturadas em profundidade foram, inicialmente, agrupados em quatro categorias: (1) construção de sentido da situação, onde foram relatadas as buscas pelo equilíbrio entre o natural e inevitável e o perturbador e desconfortável, características atribuídas à menopausa pelas mulheres; (2) repertórios interpretativos da menopausa, com relatos sobre o diálogo entre a experiência coletiva e, ao mesmo tempo, individual da menopausa; (3) recepção e oferta de conselhos, informação e apoio, o que ocorria por meio das redes formadas pelas mulheres e (4) desafios ao encontrar informação e apoio, com relatos sobre a falta de consenso entre profissionais, a dificuldade de acesso ao sistema de saúde e outros.

Os dados dessas categorias foram, então, analisados conforme o modelo proposto por McKenzie (2003). Apesar das diferenças, Yeoman (2010) considera o referido modelo bastante flexível, mas aponta a ausência de uma etapa relacionada ao uso da informação como sua principal limitação. A autora identificou que, à medida que a jornada de uma mulher pela menopausa prosseguia, ela tornava-se uma importante fonte de informação para as demais. Assim, o indivíduo buscador de informação tornava-se, também, uma fonte de 
informação e, portanto, Yeoman (2010) julgou necessário acrescentar ao modelo uma dimensão relativa ao uso da informação. Outra alteração efetuada no modelo teve origem no reconhecimento de que nem todas as barreiras podem ser superadas com sucesso. Yeoman (2010) relata que as dúvidas sobre terapias de reposição hormonal, manifestadas pelas participantes do estudo de menopausa, não puderam ser resolvidos, já que a medicina ainda não era capaz de fornecer respostas. Frente a essas limitações, Yeoman (2010) concebeu um modelo estendido, apresentado na Figura 3.

Figura 3 - Versão estendida do modelo de McKenzie.

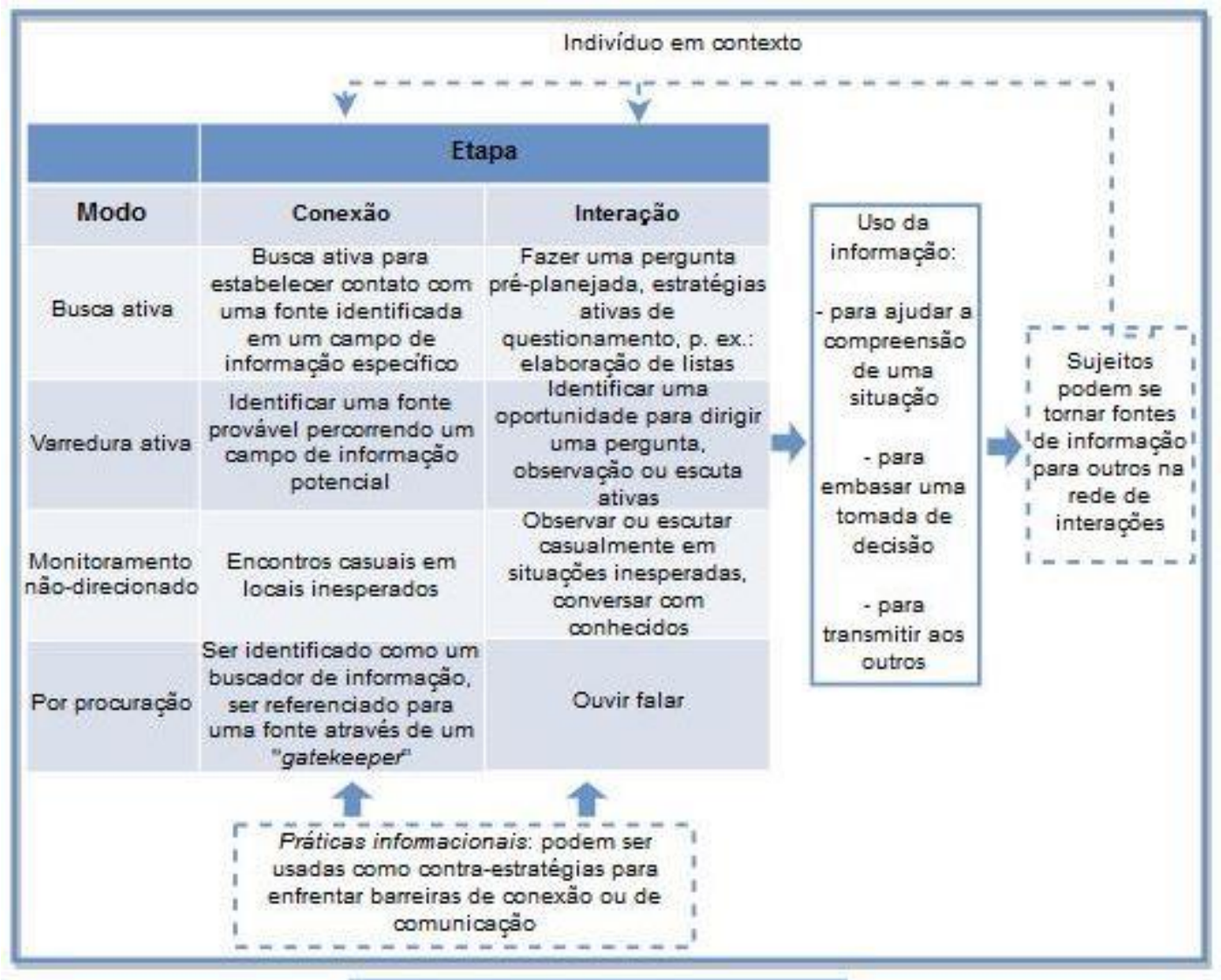

Fonte: Adaptado de Yeoman (2010, tradução nossa).

Yeoman (2010) relata que muitas práticas informacionais identificadas enquadraram-se no modelo de McKenzie (2003), entretanto a decisão de onde elas melhor se enquadrariam não foi simples. Ao contrário do estudo de McKenzie (2003), não foram identificados relatos de encontro casual de informações. Yeoman (2010) atribui esse fato à discrição das mulheres em relação à menopausa, muitas vezes não conversando tão abertamente sobre o 
assunto quanto as grávidas de gêmeos. Além disso, a gravidez de gêmeos foi apontada como "mais visível" que a menopausa, podendo ser percebida por mudanças no corpo da mulher. A autora ainda identificou que, no estudo sobre mulheres na menopausa, as práticas informacionais estavam relacionadas à busca por apoio mútuo. Já no estudo sobre gravidez, as práticas informacionais eram direcionadas à busca de informação.

\section{Modelo de práticas informacionais de adolescentes criadores de conteúdos digitais, por Mary Ann Harlan}

Harlan (2012) estudou as práticas informacionais de adolescentes norteamericanos criadores de conteúdos digitais, tais como blogs, vídeos e outros. O método utilizado foi a teoria fundamentada (grounded theory) ${ }^{2}$, segundo a qual a teoria emerge dos dados. Dados foram coletados em entrevistas semiestruturadas com onze participantes e dois anos de observações em comunidades digitais das quais eles eram membros e subsidiaram a construção de uma teoria sobre as práticas informacionais desses adolescentes.

A construção da teoria iniciou com a criação de categorias conceituais a partir dos dados coletados. Inicialmente, foram criadas cinco categorias referentes às experiências de informação (experiences of information), quatro delas com subcategorias. Conforme a autora, experiências de informação ocorrem a partir da interação dos sujeitos com a informação, em uma perspectiva na qual a informação é considerada como construída por meio de ações. Depois, foram criadas três categorias referentes às ações informacionais (information actions) dos participantes, todas elas com subcategorias. Para a autora, ações informacionais incluem atividades por vezes iterativas realizadas de diferentes formas pelos participantes durante a interação com a informação.

Em seguida, Harlan (2012) apresentou interseções entre experiências de informação e ações informacionais previamente mapeadas, considerando as comunidades de práticas nas quais tais experiências e ações ocorreram. As interseções deram origem a cinco outras categorias (e suas respectivas subcategorias) definidas pela autora como as práticas informacionais desses 
adolescentes. Assim, na teoria fundamentada construída, Harlan (2012) defende que as práticas informacionais dos participantes desenvolvem-se dentro de uma comunidade de prática e resultam da interseção entre experiências de informação e ações informacionais. Experiências de informação incorporam diferentes ações informacionais e, por serem situadas em contexto, as práticas informacionais “[...] são moldadas por estruturas sociais da comunidade e por affordances técnicos."3 (HARLAN, 2012, p. 156, tradução nossa).

Na Figura 4, as categorias e subcategorias referentes às experiências de informação, às ações informacionais e às práticas informacionais, definidas por Harlan (2012), são sintetizadas. Em seguida, as categorias são brevemente descritas conforme a concepção da autora e, então, o modelo de práticas informacionais proposto por ela é apresentado. Ressalta-se que, para maior fidelidade às idéias do texto original, optou-se por não traduzir, na Figura 4, uma das subcategorias (embodiment), um termo de difícil tradução para o português, geralmente utilizado para se referir à corporificação de algo. Esse é o sentido dado pela autora, que defende que experiências de informação podem ser corporificadas, fisicamente entendidas e experimentadas.

Quando eles [adolescentes] falavam de informação, indicavam que ela era concreta, sob a forma de conteúdo ou declarações explícitas ou ações visíveis. No entanto, eles também indicavam que a informação era internalizada, bem como, e consequentemente, invisível. Informação abarcava emoções ou respostas físicas. A teoria da prática introduziu um conceito que poderia articular essa compreensão da informação, corporificação (HARLAN, 2012, p. 77 , tradução nossa ${ }^{4}$ ). 


\section{Ceñestán}

Modelos de práticas informacionais

Janicy Aparecida Pereira Rocha, Adriana Bogliolo Sirihal Duarte, Claudio Paixão Anastácio de Paula

Figura 4 - Síntese das categorias e subcategorias referentes a experiências de informações, ações informacionais e práticas informacionais.

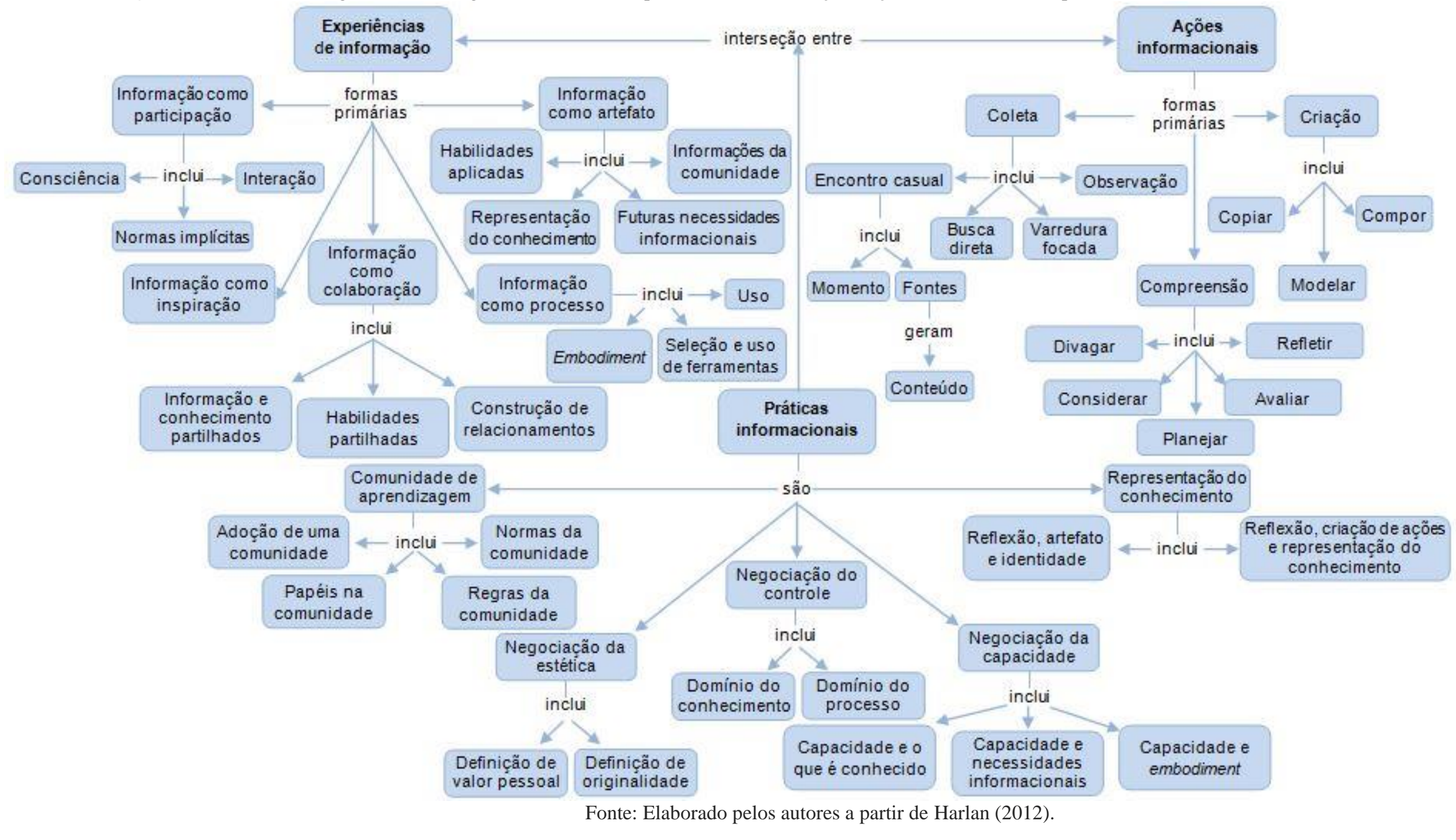


Experiências de informação acontecem de cinco formas, nas quais a informação é experimentada: (1) participação; (2) inspiração; (3) colaboração; (4) processo e (5) artefato (HARLAN, 2012). Informação como participação (information as participation) resulta da interação social dentro de uma comunidade permeada por regras e normas, com vistas a obter conhecimento sobre a comunidade e suas características. Informação como inspiração (information as inspiration) é experimentada no cotidiano, muitas vezes com ideias que surgem por acaso. Informação como colaboração (information as colaboration) resulta da produção de conteúdo por meio de habilidades e conhecimentos compartilhados. Informação como processo (information as process) está relacionada ao ato de criar conteúdo, bem como ao conhecimento e domínio de ferramentas disponíveis. Informação como artefato (information as artifact) refere-se ao conteúdo produzido, tais como vídeos, músicas, artes visuais, e ao conhecimento abstrato, como regras implícitas da comunidade.

Harlan (2012) identificou três formas de ações informacionais: (1) coleta (gathering); (2) compreensão (thinking) e (3) criação (creating). A coleta de informação ocorre por meio de atividades como busca direta, encontro casual e outras. A compreensão ocorre por meio de atividades como reflexão, avaliação e outras. Já a criação da informação inclui atividades como cópia, modelagem e composição. A partir da interseção entre experiências de informação e ações informacionais, cinco práticas informacionais foram identificadas: (1) comunidade de aprendizagem (learning comunity); (2) negociação da estética (negotiating aesthetic); (3) negociação de controle (negotiating control); (4) negociação de capacidade (negotiating capacity) e (5) representação do conhecimento (representing knowledge) (HARLAN, 2012). Tais práticas e suas relações são representadas no modelo de práticas informacionais dos adolescentes criadores de conteúdos digitais, apresentado na Figura 5. 
Figura 5 - Modelo de práticas informacionais de adolescentes criadores de conteúdos digitais.

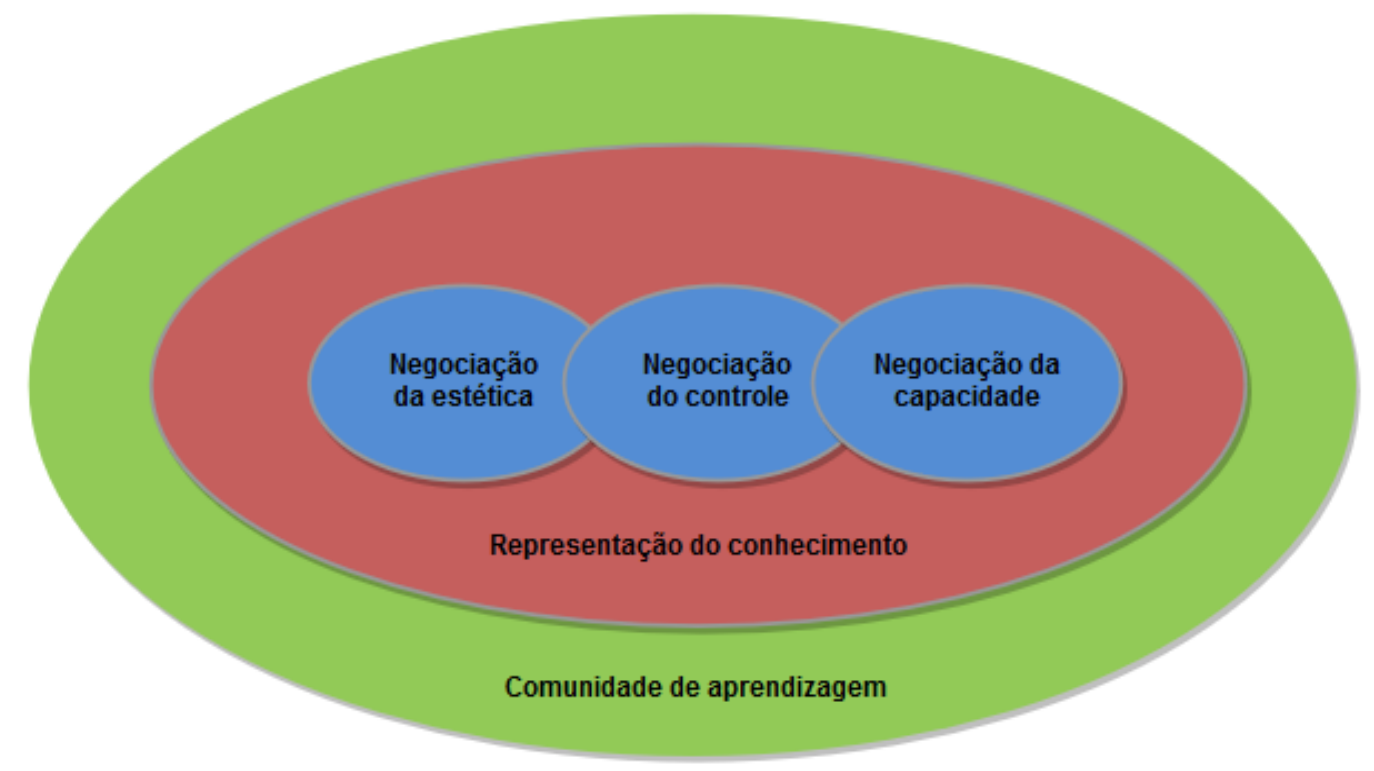

Fonte: Adaptado de Harlan (2012, tradução nossa).

A comunidade de aprendizagem permite que o adolescente construa seu entendimento sobre a comunidade, decida engajar-se nela ou não, posicione-se dentro dos papéis da comunidade e crie conteúdo conforme as regras implícitas e explícitas da comunidade. Por intermédio da negociação da estética, o adolescente define a originalidade e o valor de determinado conteúdo ou ambiente informacional. Por meio da negociação de controle, os adolescentes constroem um entendimento do conhecimento existente na comunidade através da interação com outros integrantes. Por meio da negociação de capacidade, os adolescentes aplicam informações e habilidades na construção de novos conteúdos que, posteriormente, são compartilhados. A representação do conhecimento é resultado das negociações da estética, do controle e da capacidade. Por meio dessas práticas, portanto, os adolescentes criam conteúdos influenciados por suas identidades.

Conforme Harlan (2012), o modelo representa a interdependência das cinco práticas informacionais identificadas. A comunidade de aprendizagem é representada em um círculo maior porque é a partir dela que os adolescentes conhecem a comunidade e decidem engajar-se nela para, então, desenvolver as demais práticas. As práticas relacionadas à negociação estão sobrepostas, visto que podem ocorrer simultaneamente. Por exemplo, a negociação da estética 
também acontece quando os adolescentes negociam o controle da informação que encontram. A negociação de capacidade acontece quando os adolescentes negociam suas habilidades para criar conteúdo. As três práticas de negociação contribuem para a representação do conhecimento e essa contribui para a comunidade de aprendizagem, uma vez que o conhecimento representado contém informações sobre a comunidade na qual foi compartilhado.

\section{Considerações finais}

Todos os modelos de práticas informacionais aqui apresentados adotam uma abordagem socioconstrucionista, segundo a qual as atividades informacionais são executadas por sujeitos ativos que constroem a informação a partir de interações sociais situadas em comunidades. Embora os três modelos tenham sido desenvolvidos conforme o conceito de busca de informação na vida cotidiana, eles têm origens em ambientes diferentes e, portanto, não descrevem o mesmo conjunto de atividades, nem consideram os mesmos elementos constituintes e/ou influenciadores das práticas informacionais dos sujeitos estudados. Apesar disso, os modelos também apresentam algumas características em comum.

Os modelos de Mckenzie (2003) e Yeoman (2010) são bastante parecidos, o que se deve ao fato de o segundo ser uma versão estendida do primeiro. Apesar de ambos originarem-se de estudos relacionados à busca de informação cotidiana, as situações nas quais tais buscas ocorreram são diversas e têm semelhanças e diferenças significativas. A principal semelhança é que ambos os estudos abordam mulheres vivendo situações únicas: a primeira gravidez de gêmeos e a menopausa. Ambas são situações singulares nas quais as mulheres estão susceptíveis a vivenciar desafios e atitudes semelhantes (YEOMAN, 2010). Entretanto, conforme Yeoman (2010) uma situação difere, substancialmente, da outra de várias formas: (1) culturalmente, a menopausa é vista negativamente, sendo associada ao envelhecimento; já a gravidez de gêmeos é vista de forma positiva, sendo associada à fertilidade; (2) a gravidez dura menos tempo que a menopausa e pode ser notada pela aparência física da 
mulher; (3) a maioria das mulheres passa pela menopausa, mas não pela gravidez de gêmeos.

Essas diferenças refletem nas práticas informacionais identificadas em ambos os grupos. Por exemplo, o estudo de Yeoman (2010) não identificou o encontro casual de informações, tão relatado pelas participantes do estudo de McKenzie (2003). A autora sugere que isso pode acontecer pela discrição das mulheres em relação à menopausa, que não é perceptível como a gravidez de gêmeos. Yeoman (2010) julgou necessário incluir elementos como interação e uso da informação devido a características específicas do ambiente analisado, no qual as participantes interagiam entre si, uma sendo fonte de informação para outra e vice-versa. Talvez isso aconteça porque o período de menopausa é mais extenso que o da gravidez. Por outro lado, acredita-se que o mesmo seja válido para mulheres grávidas; entretanto, a pesquisa de Mckenzie (2003) optou por não considerar as participantes como fontes de informação, considerando-as apenas como buscadoras.

Já o estudo de Harlan (2012), embora também relacionado à busca de informação cotidiana, foi desenvolvido em um ambiente bastante diferente dos demais e, consequentemente, identifica atividades e elementos constituintes diferentes. Todavia, elementos semelhantes também foram identificados, tais como encontro casual de informação, uso da informação, interação e colaboração entre os sujeitos e outros. Percebe-se, também, que tal modelo aborda o compartilhamento de informação - por meio da representação do conhecimento - de forma mais abrangente que o modelo de Yeoman (2010). A busca e o uso da informação visam à criação de novos conhecimentos pelos integrantes da comunidade e seu compartilhamento com os demais integrantes.

Ao considerar a abrangência dos modelos, o de McKenzie (2003) mostrase mais restrito por se concentrar apenas na etapa de busca de informação. Já o modelo de Yeoman (2010) considera a etapa de busca de informação de forma similar ao modelo de McKenzie (2003), mas também acrescenta uma dimensão referente ao uso da informação. Sendo assim, sua proposta de modelo estendido aproxima-se mais da noção de práticas informacionais como conceito guardachuva (SAVOLAINEN, 2007), por descrever não apenas os fenômenos 
referentes à busca, mas também ao uso e compartilhamento da informação. Embora Yeoman (2010) não tenha nomeado uma dimensão de compartilhamento em seu modelo, ela surge de forma implícita quando a autora afirma que os sujeitos buscadores de informação podem tornar-se fontes de informação para os demais na rede de interações.

O modelo de Harlan (2012) inclui as três etapas relacionadas aos fenômenos informacionais por Savolainen (2007). Para se tornarem integrantes de uma comunidade digital, os adolescentes buscam informações sobre ela e a usam para decidir inserirem-se na comunidade ou não. Já inseridos, eles continuam buscando e usando informação, mas também passam a produzir e compartilhar informação dentro daquela comunidade. As formas como os participantes buscam, usam e compartilham informações são exploradas de forma mais aprofundada por Harlan (2012) quando ela identifica as interseções entre experiências de informação e ações informacionais.

Embora a representação gráfica do modelo proposto por essa autora seja mais simples se comparada a dos modelos de McKenzie (2003) e de Yeoman (2010), a análise descritiva do modelo é bastante ampla. Isso pode ser justificado pelo próprio objetivo do trabalho: fazer com que uma teoria emergisse dos dados. Para tal, a referida autora empreende um grande esforço em identificar e descrever as experiências de informação e as ações informacionais dos adolescentes criadores de conteúdos. Em seguida, a autora apresenta um modelo (Figura 5) no qual apenas as práticas informacionais dos adolescentes criadores de conteúdos e as relações entre elas são representadas. Então, o modelo proposto é descrito de forma que a origem de cada prática informacional é apresentada a partir de interseções entre as experiências de informação e as ações informacionais previamente identificadas e descritas.

É preciso atentar-se ao fato de que o quadro conceitual fornecido por esses modelos pode não cobrir a totalidade dos fenômenos possíveis nos contextos aos quais eles pertencem. Fenômenos identificados, mas não considerados relevantes pelos autores podem não ter sido incluídos da mesma forma que alguns fenômenos podem não ter sido identificados por não fazerem parte dos objetivos do autor ou de sua visão de mundo. Todos os três modelos 
são constituídos por um conjunto de categorias que emergiram a partir dos dados das pesquisas e das relações entre elas. Entretanto, em nenhum deles, tais categorias devem ser entendidas como sequenciais, como ocorre em muitos modelos de comportamento informacional. Esses modelos apresentam apenas relações sistemáticas entre os conceitos, porém, essa sistematização objetiva a organização, não devendo remeter à ideia de etapas sucessivas, mas de etapas que podem ocorrer em ordens diferentes daquelas representadas nos modelos.

\section{Agradecimentos}

À FAPEMIG pelo aporte financeiro ao projeto de tese do qual se origina esse artigo. Aos integrantes do Grupo de Pesquisa em Práticas Informacionais do PPGCI/UFMG pelas discussões e reflexões propiciadas.

\section{Referências}

ARAÚJO, Carlos Alberto Ávila. O sujeito informacional no cruzamento da Ciência da Informação com as Ciências Sociais. In: ENCONTRO NACIONAL DE PESQUISA EM CIÊNCIA DA INFORMAÇÃO, 14., 2013, Florianópolis. Anais... Florianópolis: ENANCIB, 2013.

ARAÚJO, Carlos Alberto Ávila. Imaginação e sociabilidade: novos conceitos para o estudo de usuários da informação. In: ENCONTRO NACIONAL DE PESQUISA EM CIÊNCIA DA INFORMAÇÃO, 16., 2015, João Pessoa. Anais... João Pessoa: ENANCIB, 2015.

BATISTA, Irinéa de Lourdes; SALVI, Rosana Figueiredo; LUCAS, Lucken Bueno. Modelos científicos e suas relações com a epistemologia da ciência e a educação científica. In: ENCONTRO NACIONAL DE PESQUISA EM EDUCAÇÃO EM CIÊNCIAS, 8., 2011, Campinas. Anais... Campinas: ENPEC, 2011.

BOURDIEU, Pierre. Distinction: a social critique of the judgement of tast. London: Doutledge, 1984.

BØYUM, Idunn; AAB $\varnothing$, Svanhild. The information practices of Business $\mathrm{PhD}$ students. New Library World, Bingley, v. 116, n. 3/4, p. 187-200, 2015.

COIMBRA, Maria Leonor Valfigueira. Uma perspectiva informacional sobre o laboratório: estudo das práticas informacionais dos físicos experimentais de partículas. 2008. Dissertação (Mestrado em Estudos de Informação e Bibliotecas 
Digitais) - Departamento de Ciências e Tecnologias da Informação, Instituto Superior de Ciências do Trabalho e da Empresa, Lisboa, 2008.

FURTADO, Renata Lira; ALCARÁ, Adriana Rosecler. Modelos de comportamento informacional: uma análise de suas características. In: SEMINÁRIO CIENTÍFICO ARQUIVOLOGIA E BIBLIOTECONOMIA, 4., 2015, Marília. Anais... Marília: UNESP, 2015.

GOUVEIA JÚNIOR, Amauri. O conceito de modelo e sua utilização nas ciências do comportamento: breves notas introdutórias. Revista Estudos de Psicologia, Campinas, v. 16, n. 1, p. 13-16, 1999.

HARLAN, Mary Ann. Information practices of teen content creators: the intersection of action and experiences. A Grounded Theory study. 2012. Thesis (Doctor of Philosophy) - School of Information Systems, Science and Engineering Faculty, Queensland University of Technology, Queensland, Austrália, 2012. Disponível em:

<http://eprints.qut.edu.au/57125/1/Mary_Harlan_Thesis.pdf>. Acesso em: 16 fev. 2016.

ISAH, Esther Ebole. Physicians' information practices: a case study of a medical team at a Teaching Hospital. 2009. Thesis (Doctor of Philosophy in Library and Information Science) - Swedish School of Library and Information Science, University of Borås, Borås, Suécia, 2009. Disponível em: $\langle$ https://www.diva-portal.org/smash/get/diva2:877020/FULLTEXT01.pdf〉. Acesso em: 16 ago. 2015.

MARTINEZ-SILVEIRA, Martha; ODDONE, Nanci. Necessidades e comportamento informacional: conceituação e modelos. Ciência da Informação, Brasília, v. 36, n. 2, p. 118-127, 2007. Disponível em: <http://www.scielo.br/scielo.php?script=sci_arttext\&pid=S0100-

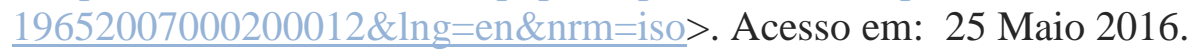

MCKENZIE, Pamela J. A model of information practices in accounts of everyday-life information seeking. Journal of Documentation, Bingley, v. 59, n. 1, p. 19-40, 2003.

NUNES, Jefferson Veras. Vivência em rede: uma etnografia das práticas sociais de informação dos usuários de redes sociais na internet. 2014. Tese (Doutorado em Ciência da Informação) - Faculdade de Filosofia e Ciências, Universidade Estadual Paulista Júlio de Mesquita Filho, Marília, 2014.

OLIPHANT, Tami. The information practices of people living with depression: constructing credibility and authority. 2010. Thesis (Doctor of Philosophy) - Faculty of Information and Media Studies, The School of Graduate and Postdoctoral Studies, University of Western Ontario, London, Ontario, Canada, 2010. Disponível em: 
$\langle$ http://ir.lib.uwo.ca/cgi/viewcontent.cgi?article=1049\&context=etd $>$. Acesso em: 12 mar. 2016.

SAYÃO, Luís Fernando. Modelos teóricos em ciência da informação; abstração e método científico. Ciência da Informação, Brasília, v. 30, n. 1, p. 82-91, 2001.

SAVOLAINEN, Reijo. Everyday life information seeking: approaching information seeking in the context of "way of life". Library \& Information Science Research, Amsterdam, v. 17, n. 3, p. 259-294, 1995.

SAVOLAINEN, Reijo. Everyday life information seeking. In: FISHER, Karen.; ERDELEZ, Sanda; McKECHNIE, Lynne. (Eds.). Theories of information behavior. Medford: Information Today Inc, 2006. p. 143-148.

SAVOLAINEN, Reijo. Information behavior and information practice: reviewing the "umbrella concepts" of information-seeking studies. Library Quarterly, Chicago, v. 77, n. 2, p. 109-132, 2007.

SAVOLAINEN, Reijo. Conceptualizing information need in context. Information Research, Lund, v. 17, n. 4, 2012. Disponível em: <http://www.informationr.net/ir/17-4/paper534.html\#.WBC8zSREzDc>. Acesso em: 14 fev. 2016.

TALJA, Sanna. The domain analytic approach to scholar's information practices. In: FISHER, Karen; ERDELEZ, Sanda; MCKECHNIE, Lynne (Eds.). Theories of Information Behavior. Medford: Information Today, 2005. p. 123127.

WILSON, Thomas Daniel; SAVOLAINEN, Reijo. The behaviour/practice debate: a discussion prompted by Tom Wilson's review of Rejio Savolainen's, "Everyday information practices: a social phenomenological perspective". Information Research, Lund, v. 14, n. 2, 2009. Disponível em: <http://www.informationr.net/ir/14-2/paper403.html>. Acesso em: 28 jan. 2016.

YEOMAN, Alison. Applying McKenzie's model of information practices in everyday life information seeking in the context of the menopause transition. Information Research, Lund, v. 15, n. 4, 2010. Disponível em: <http://InformationR.net/ir/15-4/paper444.html>. Acesso em: 20 abr. 2015.

\title{
Models of information practices
}

\author{
Abstract: This paper presents and discusses three models of information \\ practices identified in the literature of Information Science. The models were \\ identified from searches conducted on Portal de Periódicos da Coordenação de \\ Aperfeiçoamento de Pessoal de Nível Superior and on the Brazilian Digital
}


Library of Theses and Dissertations (BDTD). All of them have been developed in the context of everyday life information seeking and adopted a social constructionist approach. Although they have some similarities, the models differ by describing different activities and by considering different elements as constituents and/or influencers of the informational practices identified.

Keywords: Information Practices. Information Behavior. Models. Everyday life. Information seeking.

Recebido: 15/08/2016

Aceito: 03/10/2016

1 Os dados da pesquisa de McKenzie (2003) foram colhidos com a combinação de duas técnicas: entrevistas semiestuturadas em profundidade com duração de 25 a 110 minutos e uma variante da técnica diário-entrevista: a pesquisadora telefonou para cada participante duas vezes durante a semana seguinte à entrevista inicial questionando sobre os incidentes ocorridos desde a última conversa.

${ }^{2}$ GLASER, Barney; STRAUSS, Anselm. The discovery of grounded theory: strategies for qualitative research. Chicago: Aldine Publishing, 1967. 271 p.

${ }^{3}$ Affordances técnicos são ferramentas de uma comunidade que permitem interações com seu conteúdo (p. ex.: comentar, usar emoticons para indicar uma reação e outras) e, portanto, produzem práticas sociais (HARLAN, 2012).

4 "When they spoke of information they indicated that information was concrete in the form of content, or explicit statements or visible actions. However, they also indicated that it was internalized as well, and consequently, invisible. Information was emotions, or physical response. Practice theory presented a concept that could articulate this understanding of information, embodiment." (HARLAN, 2012, p. 77). 